\title{
Monoclinic and triclinic phases in higher-order Devonshire theory
}

\author{
David Vanderbilt and Morrel H. Cohen \\ Department of Physics and Astronomy, Rutgers University, Piscataway, New Jersey 08854-8019
}

(November 16, 2000)

\begin{abstract}
Devonshire theory provides a successful phenomenological description of many cubic perovskite ferroelectrics such as $\mathrm{BaTiO}_{3}$ via a sixth-order expansion of the free energy in the polar order parameter. However, the recent discovery of a novel monoclinic ferroelectric phase in the PZT system by Noheda et al. (Appl. Phys. Lett. 74, 2059 (1999)) poses a challenge to this theory. Here, we confirm that the sixth-order Devonshire theory cannot support a monoclinic phase, and consider extensions of the theory to higher orders. We show that an eighth-order theory allows for three kinds of equilibrium phases in which the polarization is confined not to a symmetry axis but to a symmetry plane. One of these phases provides a natural description of the newly observed monoclinic phase. Moreover, the theory makes testable predictions about the nature of the phase boundaries between monoclinic, tetragonal, and rhombohedral phases. A ferroelectric phase of the lowest (triclinic) symmetry type, in which the polarization is not constrained by symmetry, does not emerge until the Devonshire theory is carried to twelfth order. A topological analysis of the critical points of the free-energy surface facilitates the discussion of the phase transition sequences.
\end{abstract}

PACS: 77.80.Bh, 77.84.Dy, 64.70.Kb

\section{INTRODUCTION}

Many of the most important and best studied ferroelectric materials adopt the simple-cubic perovskite structure at high temperature and undergo structural phase transitions to distorted ferroelectric structures at lower temperature. Among the best known simple compounds of this kind are $\mathrm{BaTiO}_{3}$ and $\mathrm{PbTiO}_{3}$. Upon cooling, $\mathrm{BaTiO}_{3}$ undergoes a sequence of ferroelectric transitions, first from the cubic (C) to a tetragonal (T), then to an orthorhombic $(\mathrm{O})$, and finally to a rhombohedral $(\mathrm{R})$ phase. Passing through this sequence, the polarization $\mathbf{P}$ first vanishes in the $\mathrm{C}$ phase, and then becomes oriented in the [001], [011], and [111] directions in the T, O, and $\mathrm{R}$ phases, respectively. $\mathrm{PbTiO}_{3}$ undergoes a single ferroelectric transition from the $\mathrm{C}$ to $\mathrm{T}$ phase.

In a classic 1948 paper, Devonshirel was able to explain the observed phases and phase transition sequence quite naturally in terms of a phenomenological Landautype expansion of the free energy in terms of the ferroelectric order parameter $\mathbf{P}$. Making use of cubic symmetry and truncating the expansion to sixth order in $\mathbf{P}$, Devonshire was able to arrive at a simple model with only a single temperature-dependent second-order coefficient, and only three temperature-independent higherorder coefficients. The polarization $\mathbf{P}$ is the primary order parameter, and the crystallographic labels ( T, R, etc.) refer to the distortions induced by the polarization and the resulting strain. Despite its simplicity, this model could successfully reproduce the phase-transition sequence 1 and the piezoelectric and other properties of $\mathrm{BaTiO}_{3}$. With a simple modification of the anharmonic coefficients, the qualitative behavior of $\mathrm{PbTiO}_{3}$ could be equally well reproduced.

However, the material that is currently in most widespread use for piezoelectric transducer and related ap- plications is the solid solution $\mathrm{PbZr}_{1-x} \mathrm{Ti}_{x} \mathrm{O}_{3}$, commonly known as PZT. The standard understanding of the phase diagram of PZT has been as follows 3 PZT undergoes a transition from the simple cubic $\mathrm{C}$ phase to a ferroelectric phase at a Curie temperature $T_{c}$ that ranges from about $490^{\circ} \mathrm{C}$ at $x=1$ to $230^{\circ} \mathrm{C}$ at $x=0$. The transition occurs to the $\mathrm{T}$ phase for $x$ greater than about 0.48 , and to the $\mathrm{R}$ phase for smaller $x$. At $T<150^{\circ} \mathrm{C}$ or $x<0.1$, some more complex phases involving antiferroelectric (AFE) or antiferrodistortive (AFD, i.e., involving rotations of oxygen octahedra) displacements also occur. The phase boundary between the simple $\mathrm{T}$ and $\mathrm{R}$ phases, known as the morphotropic phase boundary (MPB), is an almost verfical line in the $x-T$ plane at about $x=0.48$. Haun et al. thave successfully extended the Devonshire model to the case of PZT by including the AFE and AFD degrees of freedom in the phenomenological free energy, still including only terms up to sixth order overall. This model successfully described the simple $\mathrm{R}-\mathrm{T}$ transition that was understood to occur below $T_{c}$ at the MPB.

As it happened, a surprise was in store. By working with highly purified and carefully prepared samples of PZT, Noheda and coworkers have recently shown 8 that a sliver of monoclinic (M) phase actually interposes itself between the $\mathrm{R}$ and $\mathrm{T}$ phases in a very narrow composition range (of order $3-4 \%$ in $x$ ). That is, at least below $\sim 100^{\circ} \mathrm{C}$, the transition is first from $\mathrm{T}$ to $\mathrm{M}$ at an $x_{c 2}$ between about 0.48 and 0.51 (depending on temperature), and then from $\mathrm{M}$ to $\mathrm{R}$ at $x_{c 1} \simeq 0.47$, with decreasing $x$. The orientation of $\mathbf{P}$ is, respectively, along [001], [uuv] $(u<v)$, and [111] in the $\mathrm{T}, \mathrm{M}$, and $\mathrm{R}$ phases, respectively. The experiments have not yet clarified whether or not a direct $\mathrm{T}-\mathrm{R}$ transition occurs in the higher temperature range $100^{\circ} \mathrm{C}<T<T_{c}$, or whether the sliver of $\mathrm{M}$ phase instead persists up to the Curie temperature $T_{c} \simeq 370^{\circ} \mathrm{C}$. Neither the Devonshire theory,î nor 
the modification of Haun et al. 1 predicted the possible occurrence of the $\mathrm{M}$ phase. On the other hand, simulations based on a first-principles effective Hamiltonian approach 9 have very recently provided confirmation of the existence of the M phase in just the observed composition range. 10

We make a brief aside to establish notation. In the $\mathrm{C}$ phase (space group $P m 3 m), \mathbf{P}=\mathbf{0}$. When $\mathbf{P}$ is constrained to a symmetry axis lying along [001], [111], or [011], the resulting phase and space-group labels become $\mathrm{T}(P 4 m m), \mathrm{R}(R 3 m)$, or O $(A m m 2)$, respectively. Similarly, the $\mathrm{M}$ phases arise when $\mathbf{P}$ is confined to a mirror plane. We can distinguish three cases: $\mathrm{M}_{\mathrm{C}}(P m)$, in which $\mathbf{P}$ is along [0uv]; and $\mathrm{M}_{\mathrm{A}}$ and $\mathrm{M}_{\mathrm{B}}$ (both $\mathrm{Bm}$, sometimes also denoted $\mathrm{Cm}$ ) in which $\mathbf{P}$ lies along [uuv], with $u<v$ and $u>v$, respectively. The newly observed phase of PZT is of type $\mathrm{M}_{\mathrm{A}}$. 6 \& If $\mathbf{P}$ is unconstrained by symmetry, strain coupling leads to a triclinic phase $(P 1)$. This exhausts the possible reduced-symmetry states of a cubic perovskite crystal generated by the emergence a single non-zero ferroelectric order parameter, although other phases can occur if AFE and AFD distortions are also present.

Relatively few examples are known of low-symmetry ferroelectrics in which $\mathbf{P}$ is only constrained to a symmetry plane, or in which $\mathbf{P}$ is unconstrained by symmetry. The discovery of the $M_{\mathrm{A}}$ phase in a cubic perovskite is thus of considerable note, even aside from the fact that it had been missed for so long and aside from the potential importance of this phase for understanding the large piezoelectric response in PZT.

The failure of the phenomenological theories of Devon-

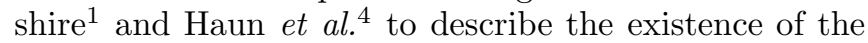
observed $\mathrm{M}_{\mathrm{A}}$ phase raises an interesting question: What is the simplest and most natural phenomenological model that does predict such a monoclinic phase? Recent work of Souza Filho et al 11 confirms that the sixth-order Devonshire expansion does not allow for the occurrence of a monoclinic phase and suggests instead a model in which the vanishing of a shear elastic constant at the critical temperature drives the transition to the $\mathrm{M}_{\mathrm{A}}$ phase. However, this requires the introduction of an additipnal instability that is unrelated to the ferroelectric one 12 That this is unnecessary is demonstrated by the work of Bellaiche, García and Vanderbilt. 10 In their simulations, the shear moduli are taken as temperature-independent, and yet the transition to the monoclinic phase still occurs, driven by the tilting of the ferroelectric polarization away from the symmetry axis.

Suspecting instead that the problem is simply related to the truncation of the expansions of Devonshiret and Haun et al. 1 to sixth order, we consider the addition of terms of eighth and higher order to the Devonshire model. The following questions then arise. At what order in the expansion do monoclinic phases first appear in the phase diagram? And, for that matter, at what order do triclinic phases first appear?

The phase diagram of such a model consists of fields in the parameter space of the model (labeled C, T, O, $\mathrm{R}, \mathrm{M}$, etc.) within which the order parameter has the specified symmetry (that is, the absolute minimum of the free energy occurs at a point in the order-parameter space of that symmetry). On a trajectory in the phase space which crosses a phase boundary, that global minimum can change into a local minimum, a saddle point, or a maximum, or simply disappear. Understanding how the set of critical or stationary points of the free energy (its minima, saddle points, and maxima in the orderparameter space) varies with the parameters of the model can thus be an important aid in understanding the phase diagram. So, in addition to searches for the global minima by direct computation, we use here a topological analysis of the Devonshire theory to elucidate the criticalpoint structure of the free energy and answer the above questions.

We show here that the simplest extension of the Devonshire theory, to eighth order in the ferroelectric order parameter, naturally admits all three kinds $\left(\mathrm{M}_{\mathrm{A}}, \mathrm{M}_{\mathrm{B}}, \mathrm{M}_{\mathrm{C}}\right)$ of monoclinic phases. Moreover, the eighth-order model makes specific and testable predictions for the types of phase boundaries that can occur. For example, it predicts that the $\mathrm{R}-\mathrm{M}_{\mathrm{A}}$ and $\mathrm{T}-\mathrm{M}_{\mathrm{A}}$ transitions should be of first and second order, respectively. On the other hand, we find that the model has to be extended all the way to twelfth order in order to describe a triclinic ferroelectric phase.

We therefore suggest that the most natural explanation for the occurrence of the $\mathrm{M}_{\mathrm{A}}$ phase in PZT is that the free-energy surface is unusually anharmonic in this material, such that the eighth-order terms play an important role. It is not difficult to speculate why this might be the case. First, PZT is a disordered material; averaging over the chemical disorder, which plays the role of a quenched random field, may tend to generate higher orders in the phenomenological energy expansion. Second, as will be discussed in Sec. V, there is considerable evidence that the ferroelectric transitions in PZT have a strong order-disorder character; mapping onto a displacive picture may then also tend to generate higherorder terms. And finally, of course, thermal fluctuations and coupling to strain may play some role. In any case, we show below that once one accepts this simple hypothesis of the importance of eighth-order terms in the freeenergy expansion, then the beharior of the moneglinic phase observed in experiments 50.8 and simulations 10 can be understood quite naturally.

This paper is organized as follows. Section II establishes the notation used for the expansion of the free energy, and reviews the symmetry considerations that lead to a simplified form of this expansion. The rules governing the numbers and types of stationary points that may occur in the order-parameter space are reviewed in Sec. III. The behavior of the models obtained by truncating the expansion at higher and higher order (fourth, sixth, eighth, tenth, and twelfth) are then carefully elucidated in Sec. IV. Section V gives a brief discussion of 
a microscopic model in which the $\mathrm{T}$ and $\mathrm{R}$ phases can be regarded as arising from fluctuations among neighboring, symmetry-equivalent local $\mathrm{M}_{\mathrm{A}}$ states. Finally, we conclude with a brief summary and discussion of future prospects in Sec. VI.

\section{FORMALISM}

We consider the case of a structural phase transition governed by a single continuous vector order parameter $\mathbf{u}$, such that the free energy

$$
F\left(\mathbf{u}, \sigma_{i}, T\right)=E\left(\mathbf{u}, \eta_{i}, S\right)-\sum_{i} \sigma_{i} \eta_{i}-T S
$$

is symmetric with respect to operations of the cubic point group. Here $\sigma_{i}$ and $\eta_{i}$ are the stress and strain tensors in Voigt notation, and $T$ and $S$ are temperature and entropy, respectively. We have in mind primarily the case in which $\mathbf{u}$ is the ferroelectric polarization $\mathbf{P}$ in a member of the cubic perovskite class, such as $\mathrm{BaTiO}_{3}$ or PZT, but the use of the symbol $\mathbf{u}$ serves as a reminder that the formalism applies to a variety of other cases as well. For a crystal with stress-free boundary conditions at some given temperature, we can expand

$$
\begin{aligned}
F(\mathbf{u}) & =C_{000}+C_{200}\left(u_{x}^{2}+u_{y}^{2}+u_{z}^{2}\right)+C_{400}\left(u_{x}^{4}+u_{y}^{4}+u_{z}^{4}\right) \\
& +C_{220}\left(u_{x}^{2} u_{y}^{2}+u_{x}^{2} u_{z}^{2}+u_{y}^{2} u_{z}^{2}\right)+C_{600}\left(u_{x}^{6}+u_{y}^{6}+u_{z}^{6}\right) \\
& +C_{420}\left(u_{x}^{4}\left[u_{y}^{2}+u_{z}^{2}\right]+u_{y}^{4}\left[u_{x}^{2}+u_{z}^{2}\right]+u_{z}^{4}\left[u_{x}^{2}+u_{y}^{2}\right]\right) \\
& +C_{222} u_{x}^{2} u_{y}^{2} u_{z}^{2}+\ldots,
\end{aligned}
$$

where terms up to sixth order in $u$ have been written explicitly, and the coefficients have been renormalized to subsume the couplings to strain.

For the ferroelectric phases we simplify matters further by focusing on the energy as a function of the orientation of the vector order parameter and introducing the function

$$
G(\hat{\mathbf{u}})=\min _{\mathbf{u} \| \hat{\mathbf{u}}} F(\mathbf{u})
$$

Thus, $G(\hat{\mathbf{u}})$ represents the ground-state energy subject to the constraint that the order parameter has given orientation. In most cases of interest, it is reasopable to expect that $G(\hat{\mathbf{u}})$ will be a smooth function of $\hat{\mathbf{u}} 13$ In this case, and suppressing the uninteresting constant term in the expansion, it follows that

$$
\begin{aligned}
G(\hat{\mathbf{u}})= & a_{4} g_{4}+a_{6} g_{6}+a_{8} g_{4}^{2} \\
& +a_{10} g_{4} g_{6}+a_{12} g_{4}^{3}+a_{12}^{\prime} g_{6}^{2}+\ldots,
\end{aligned}
$$

where

$$
\begin{aligned}
& g_{4}(\hat{\mathbf{u}})=x^{2} y^{2}+x^{2} z^{2}+y^{2} z^{2} \\
& g_{6}(\hat{\mathbf{u}})=x^{2} y^{2} z^{2}
\end{aligned}
$$

TABLE I. Values of the cubic invariant functions defined in Eqs. (5) and (4), evaluated at tetragonal (T), rhombohedral $(\mathrm{R})$, and orthorhombic $(\mathrm{O})$ orientations of the order parameter $\hat{\mathbf{u}}$.

\begin{tabular}{cccc}
\hline \hline$F(\hat{\mathbf{u}})$ & $F(\mathrm{~T})$ & $F(\mathrm{R})$ & $F(\mathrm{O})$ \\
\hline$g_{4}$ & 0 & $1 / 3$ & $1 / 4$ \\
$g_{6}$ & 0 & $1 / 27$ & 0 \\
$f_{4}$ & 0 & 4 & 3 \\
$f_{6}$ & 0 & 0 & 1 \\
$f_{8}$ & 0 & 0 & 0 \\
\hline \hline
\end{tabular}

and $\hat{\mathbf{u}}=(x, y, z)$ with $x^{2}+y^{2}+z^{2}=1$. All independent, symmetry-allowed terms up to twelfth order are explicitly given in Eq. (4); higher orders will not be needed here.

The particular form of the cubic invariants appearing in Eq. (4) is largely arbitrary. For example, one could use the "kubic harmonics" 14 instead. In Sec. IV D, we shall make use of the expansion of Eqs. (4 5) above. However, for the presentation of numerical results to be given in Sec. IV C, we find it more convenient instead to use the expansion

$$
G(\hat{\mathbf{u}})=c_{4} f_{4}+c_{6} f_{6}+c_{8} f_{8}+\ldots,
$$

where

$$
\begin{aligned}
& f_{4}(\hat{\mathbf{u}})=12 g_{4} \\
& f_{6}(\hat{\mathbf{u}})=4 g_{4}-36 g_{6} \\
& f_{8}(\hat{\mathbf{u}})=48 g_{4}^{2}-12 g_{4}-36 g_{6} .
\end{aligned}
$$

Aside from normalization constants, which are chosen to make the range of each function roughly of order unity, this choice can be uniquely defined by the following requirements. (i) The invariant $f_{n}$ contains no terms of order higher than $n$. (ii) All three invariants vanish identically for a "tetragonal" (T) value of the order parameter, e.g., $\hat{\mathbf{u}}=(100)$. (iii) $f_{6}$ and $f_{8}$ still vanish for a "rhombohedral" (R) value of the order parameter, e.g., (111) $/ \sqrt{3}$. (iv) $f_{8}$ alone vanishes for an "orthorhombic" $(\mathrm{O})$ value of the order parameter, e.g., (110)/ $\sqrt{2}$. The values of these functions evaluated at symmetry directions are summarized in Table I for later reference. Requirement (ii) just reflects an arbitrary choice of zero; requirements (iii) and (iv) simplify some later discussion. For example, phase boundaries at which $G(\mathrm{~T}), G(\mathrm{R})$, and $G(\mathrm{O})$ become degenerate in pairs are easily located (see Sec. IV ().

A few comments about the transition from $F(\mathbf{u})$ to $G(\hat{\mathbf{u}})$ in Eq. (3) are in order. First, our "reduced" theory in terms of $G(\hat{\mathbf{u}})$ can only describe the transitions among the ferroelectric phases, not the transition to the high-temperature cubic phase $(\mathbf{u}=0)$. Second, the minimization over the magnitude of $\mathbf{u}$ in Eq. (3) generally introduces higher orders into the reduced theory. For example, truncation of Eq. (2) at sixth order may still lead to terms of eighth and higher order in Eqs. (4) and (6). Thus, a statement about what kinds of critical points can occur simultaneously in an $n^{\text {th }}$-order version of the 
reduced theory does not necessarily carry over to the $n^{\text {th }}$ order version of the standard Devonshire theory. Nevertheless, a statement that a certain minimum order of expansion is needed for the existence of a certain kind of stationary point in the reduced theory does carry over to the standard Devonshire theory. 15 For example, we show later that the reduced theory must be carried to eighth order to allow for a monoclinic minimum, i.e., an equilibrium $\mathbf{u}$ lying in a $(1 \overline{1} 0)$ plane. Then the same statement applies to the standard Devonshire theory. For, suppose that $u_{0}$ is the magnitude of $\mathbf{u}$ at the minimum, and let $G_{0}(\hat{\mathbf{u}})=F\left(u_{0} \hat{\mathbf{u}}\right)$; then $G_{0}$ must have a monoclinic minimum. But $G_{0}$ is also of no higher than eighth order and so admits only the same type of minima as does $G .15$

\section{TOPOLOGICAL CONSIDERATIONS}

The rules governing the numbers and types of critical or stationary points of a scalar function defined on a continuous manifold emerge from a branch of algebraic topology called Morse theory gr, equivalently, the calculus of variations in the large.16 These rules have proved useful in analyzing lattice vibration spectra 17 and the excitation spectra of crystalline materials generally. They have not, however, enjoyed the broad application in condensed matter physics that might have been expected from these early successes.

The rules take the form of a set of inequalities and one equality. The latter is the most powerful, and we confine ourselves to considering only it explicitly. $G(\hat{\mathbf{u}})$ is an analytic function defined on the surface of a sphere, a closed two-dimensional manifold of genus zero, for which the equality becomes

$$
N_{0}-N_{1}+N_{2}=2,
$$

where $N_{0}, N_{1}$, and $N_{2}$ are the number of minima, saddle points, and maxima, respectively. This formula assumes only analyticity in $G(\hat{\mathbf{u}})$ and that its Hessian has no vanishing eigenvalues at the critical points.

Because the function $G(\hat{\mathbf{u}})$ has cubic symmetry, any stationary points that occur must be members of symmetry-related families of stationary points. Thus, it is natural to focus attention on an irreducible wedge corresponding to $1 / 48$ of the unit sphere, in terms of which Eq. (8) can be rewritten as

$$
\sum_{j} n_{j} \gamma_{j}=2
$$

where $j$ runs over the stationary points located in the interior or on the boundary of the irreducible wedge, $n_{j}$ is a degeneracy factor counting the number of images of the stationary point generated by the cubic symmetry group operations, and $\gamma_{j}$ is +1 for a maximum or minimum and -1 for a saddle point. Symmetry requires only that there be stationary points at $\mathrm{T}, \mathrm{O}$, and R; those at $\mathrm{T}$ and $\mathrm{R}$ (a)
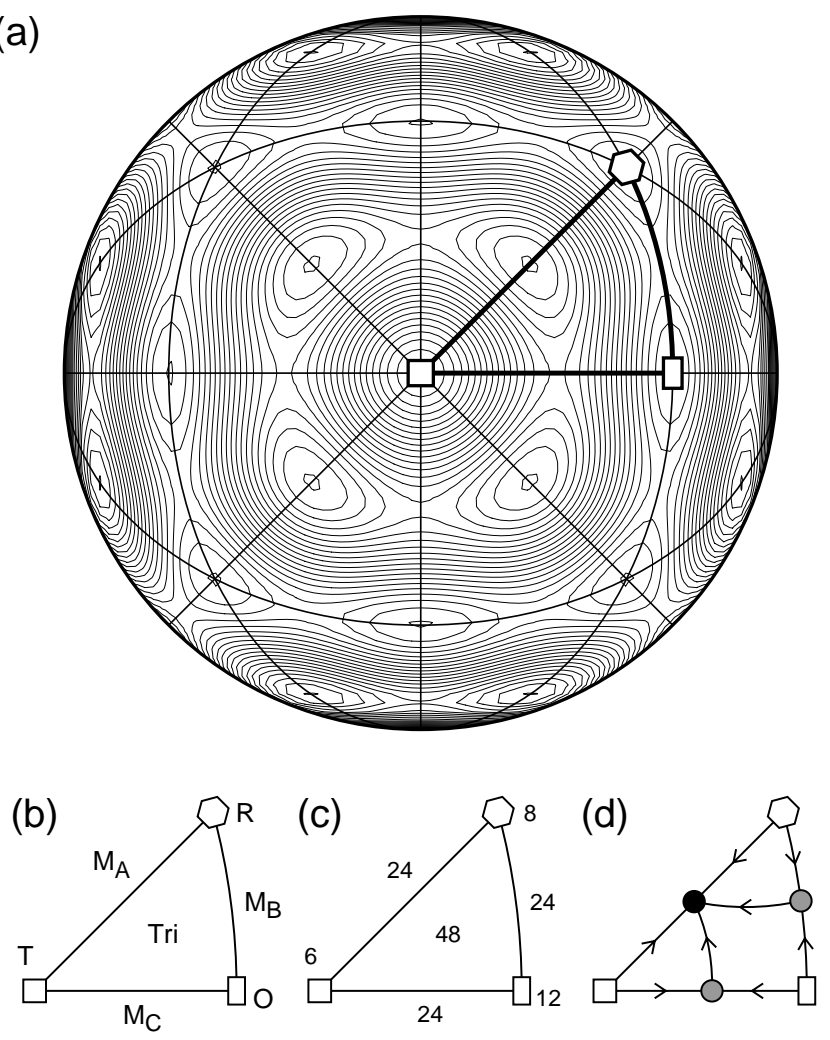

FIG. 1. (a) Contours of constant $G(\hat{\mathbf{u}})=-f_{8}(\hat{\mathbf{u}})$ on the unit sphere. (b) Labels for symmetry points and lines of the irreducible wedge. (c) Corresponding degeneracy factors $n$ indicating number of images on the full unit sphere. (d) Summary representation of the behavior of $G(\hat{\mathbf{u}})$, in which open, shaded, and filled symbols represent maxima, saddle points, and minima of $G$, respectively. Arrows indicate "downhill" flow lines.

must be maxima or minima while that at $\mathrm{O}$ can be of any type. Such a symmetry set of stationary points may or may not be large enough to satisfy the Morse relations as well. A set which contains the smallest number of critical points satisfying both the topological and symmetry requirements is denoted a minimal set.17

This type of analysis is illustrated in Fig. 1. Figure 1(a) shows an arbitrarily chosen function $G(\hat{\mathbf{u}})$ corresponding to $c_{4}=c_{6}=0$ and $c_{8}=-1$ in Eq. (6), having maxima at $\mathrm{T}, \mathrm{R}$, and $\mathrm{O}$; saddle points on the lines connecting $\mathrm{T}-$ $\mathrm{O}$ and $\mathrm{O}-\mathrm{R}$; and a minimum on the segment connecting T-R. Figure 1(b) specifies the notation that we shall use to identify the symmetry points and lines. These are chosen to correspond to the labels of distorted crystal structures (tetragonal, rhombohedral, or orthorhombic for the symmetry points; monoclinic of type 'A,' 'B,' or ' $\mathrm{C}$ ' for the symmetry lines; and triclinic for the case of $\hat{\mathbf{u}}$ pointing to the interior of the wedge). The corresponding degeneracy factors $n$ are given in Fig. 1(c). The overall behavior of the function inside the wedge is summarized in Fig. 1(d), in which the stationary points are marked by symbols that are open, shaded, or filled for maxima, 

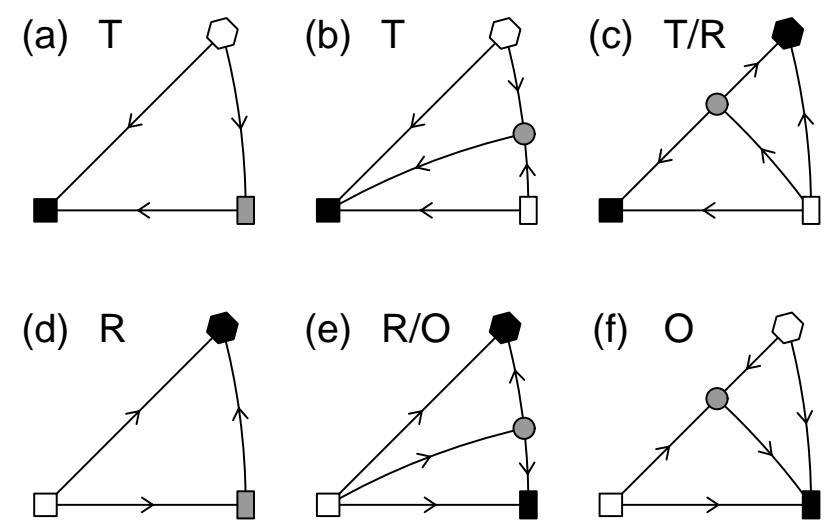

FIG. 2. Sample topologies giving rise to tetragonal (a-c), rhombohedral (c-e), and orthorhombic (e-f) phases. Open, shaded, and filled symbols represent maxima, saddle points, and minima, respectively.

saddle points, or minima, respectively. In this particular example, it can easily be seen that Eq. (9) is satisfied $(6+8+12-24-24+24=2)$.

Using Eq. (9), one can begin enumerating the possible topological diagrams for the cubic system. Figs. 2(a) and $2(\mathrm{~d})$ show the two possible diagrams in which there are only three stationary points in the irreducible wedge, the only possible minimal sets. In Fig. 2(a) the function has a unique minimum at $\mathrm{T}$, so the crystal ground state would be tetragonal. A similar situation holds for the rhombohedral case of Fig. 2(d). Figures 2(b-c) and (e-f) show four of the six configurations that can exist when there are exactly four stationary points in the wedge. (Two others, similar to 2(b) and 2(e) but with the the role reversals $\mathrm{T} \leftrightarrow \mathrm{R}$ and $\mathrm{M}_{\mathrm{B}} \leftrightarrow \mathrm{M}_{\mathrm{C}}$, are not shown.) In Fig. 2(c) there are two local minima, so that the ground state could be of type $\mathrm{T}$ or $\mathrm{R}$ depending on which minimum is deeper; a first-order transition between $\mathrm{T}$ and $\mathrm{R}$ may occur by a crossing of the minima. The simplest configuration leading to an orthorhombic ground state is shown in Fig. 2(f).

In order to arrive at configurations corresponding to monoclinic or triclinic phases, more than four stationary points are required. Figs. 3(a-c) illustrate possible configurations for monoclinic phases of type $\mathrm{A}, \mathrm{B}$, and $\mathrm{C}$, in which the order parameter lies on the $\mathrm{T}-\mathrm{R}, \mathrm{R}-\mathrm{O}$, and O-T lines, respectively. Figure 3(d) illustrates a possible triclinic phase. It is straightforward to check that Eq. (9) is satisfied for each configuration in Figs. 2 and 3.

Of course, if the expansion of Eq. (4) or (6) is truncated at a certain order, the possible types of topological behavior will be limited by the enforced "smoothness" of the functions allowed at that order. The purpose of the following Section is to explore precisely this issue, i.e., to clarify what types of phases and phase transitions can occur at each order in the expansion. With this information in hand, one can then easily determine what is the minimal model needed to study a particular physical phenomenon of interest.
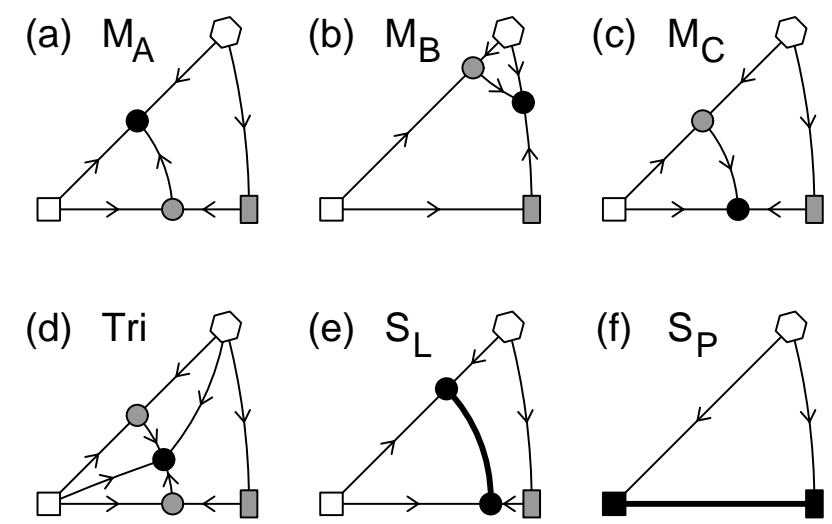

FIG. 3. (a-d) Sample topologies giving rise to monoclinic phases A, B, and C, and the triclinic phase, respectively. (e) Degenerate minimum (heavy solid curve) that can occur when $\beta=3 \pi / 4$. (f) Degenerate minimum (heavy solid line) that occurs for $\alpha=\tan ^{-1}(3)$ and $\beta=\pi$. Open, shaded, and filled symbols represent maxima, saddle points, and minima, respectively.

\section{RESULTS}

\section{A. Fourth-order theory}

If the expansion (6) is truncated at fourth order, the only non-constant cubic invariant is $f_{4}$ of Eq. (7). The $\mathrm{T}$ or R phase is favored as in Fig. 2(a) or 2(b) if $c_{4}>0$ or $c_{4}<0$, respectively. A transition between $\mathrm{T}$ and $\mathrm{R}$ phases occurs at $c_{4}=0$, but this transition is unphysical because the energy surface is perfectly flat at the transition. This degenerate behavior is an artifact of the truncation to fourth order.

\section{B. Sixth-order theory}

At sixth order in the expansion (6), the behavior is governed by the two coefficients $c_{4}$ and $c_{6}$. Clearly a common rescaling of the coefficients by a positive scale factor is irrelevant, so without loss of generality we may set $c_{4}^{2}+c_{6}^{2}=1$. It is then convenient to let

$$
c_{4}=\cos (\alpha), \quad c_{6}=\sin (\alpha) .
$$

By doing numerical calculations and making plots such as that shown in Fig. 1(a), we find that only three phases exist at this level. For $-\tan ^{-1}(3)<\alpha<\pi / 2$, the system is in the T ground state; for $\pi / 2<\alpha<5 \pi / 4$, the system is in the $\mathrm{R}$ ground state; and for $5 \pi / 4<\alpha<2 \pi-$ $\tan ^{-1}(3)$ the system is in the $\mathrm{O}$ ground state. Fig. 2 illustrates the sequence of topologies traversed as $\alpha$ is increased. Starting from $\alpha=-\tan ^{-1}(3)$, one finds $\mathrm{T}$ phases corresponding to Figs. 2(a-c) consecutively, until a first-order transition occurs to the $\mathrm{R}$ state at $\alpha=\pi / 2$. Then one finds $\mathrm{R}$ phases as illustrated in Figs. 2(c-e) until a first-order transition occurs to the $\mathrm{O}$ phase at 
$\alpha=5 \pi / 4$. Finally, O phases corresponding to Figs. 2(ef) are found as $\alpha$ is increased further up to $2 \pi-\tan ^{-1}(3)$. The transition from the $\mathrm{O}$ to the $\mathrm{T}$ phase is degenerate in the sense that the energy surface becomes exactly flat along the entire $\mathrm{M}_{\mathrm{C}}$ symmetry line at the critical $\alpha$ (this being an artifact of truncation to low order).

It is important to emphasize that that no monoclinic phase is possible in the sixth-order model. In the range $\pi+\tan ^{-1}(3 / 2)<\alpha<2 \pi-\tan ^{-1}(3)$ (i.e., $1.3128 \pi<$ $\alpha<1.6024 \pi)$ in which such a 1D local minimum appears along the $\mathrm{M}_{\mathrm{A}}$ symmetry line, it is always unstable in the second dimension (i.e., it is a saddle point), and the true minimum is at the $\mathrm{O}$ point as in Fig. 2(f).

Further details of the sequence of topologies and the boundaries between them is given in the Appendix.

\section{Eighth-order theory}

When the model of Eq. (6) is carried to eighth order, it no longer becomes profitable to enumerate every possible topology, as was done above for the sixth-order theory. Instead, we choose to focus just on the "phase diagram" that is generated by finding the ground-state symmetry as a function of the parameters $c_{4}, c_{6}$, and $c_{8}$. A common scaling of the magnitudes of these coefficients is again unimportant, so we can describe the phase diagram in terms of two dimensionless parameters that we may take as

$$
\begin{aligned}
& c_{4}=\cos (\alpha), \\
& c_{6}=\sin (\alpha) \cos (\beta), \\
& c_{8}=\sin (\alpha) \sin (\beta),
\end{aligned}
$$

where $0<\alpha<\pi$ and $0<\beta<2 \pi$. From this point of view, the "parameter space" is just the unit sphere determined by polar and azimuthal angles $\alpha$ and $\beta$ respectively.

Figure 4 shows the phase diagram that emerges from a careful numerical study of the minimization of Eq. (6) as a function of $\alpha$ and $\beta$. The plot is a mapping of the unit sphere onto the page. The points at the "north" and "south" poles $(\alpha=0$ and $\alpha=\pi)$ are the only ones accessible in the fourth-order theory (Sec. IVA); the dotted vertical lines at $\beta=0$ and $\beta=\pi$ correspond to the locus of points in parameter space that were explored by the sixth-order model (Sec. IV B).

As can be seen from Fig. 4, six of the seven possible phases (i.e., possible symmetries of a non-zero order parameter) are accessed by the eighth-order model. In addition to the $\mathrm{T}, \mathrm{R}$, and $\mathrm{O}$ phases that appeared already at sixth order, all three monoclinic phases $\left(\mathrm{M}_{\mathrm{A}}\right.$, $\mathrm{M}_{\mathrm{B}}$, and $\mathrm{M}_{\mathrm{C}}$ ) are now stable in some region of the phase diagram. However, the areas covered by the $\mathrm{M}_{\mathrm{C}}$ and especially $M_{B}$ regions are relatively small, so these phases may be harder to find in real systems than the $\mathrm{M}_{\mathrm{A}}$ phase.

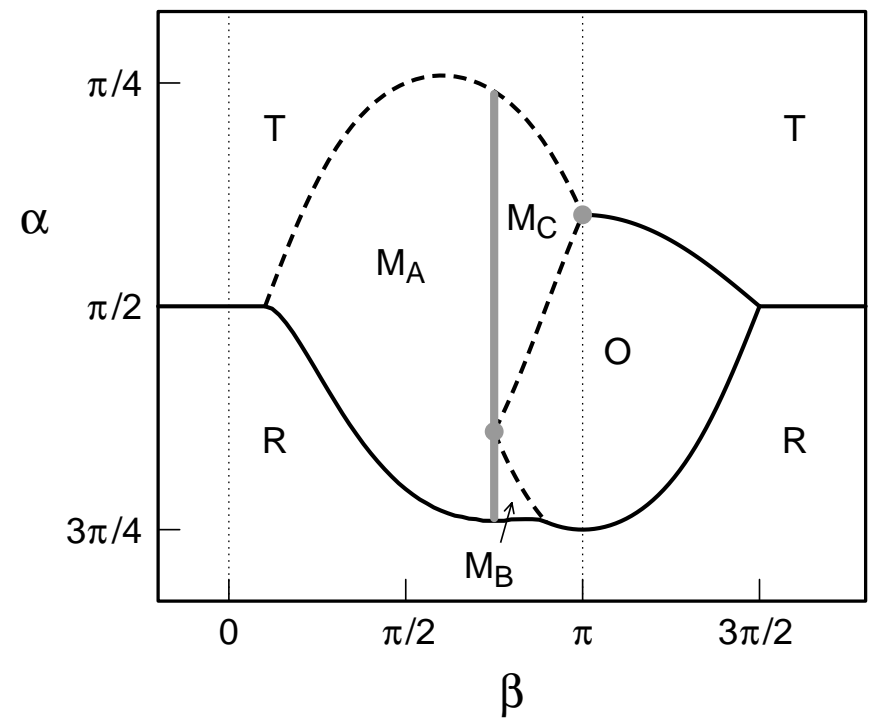

FIG. 4. Phase diagram in the space of parameters $\alpha$ and $\beta$ of the eighth-order theory as defined in Eq. (11). Solid and dashed lines are first-order and second-order phase boundaries, respectively. The vertical grey line and the grey dots indicate cases for which degenerate minima occur. Fine vertical dotted lines indicate the domain of the sixth-order theory.

Solid and dashed phase boundaries indicate transitions of first and second order, respectively, as determined numerically. According to Landau theory, there are two necessary conditions for a transition to be of second order: (i) the symmetry group of one phase must be a subset of the symmetry group of the other, $\mathcal{G} \subset \mathcal{G}_{0}$; and (ii) from the order-parameter displacements that lead from the high-symmetry to the low-symmetry phase, it should be impossible to construct a third-order invariant of $\mathcal{G}_{0} .18$ Transitions of type T-R, T-O, and $\mathrm{O}-\mathrm{R}$ are necessarily first-order because of condition (i), and transitions from the $\mathrm{R}$ phase to $\mathrm{M}_{\mathrm{A}}$ or $\mathrm{M}_{\mathrm{B}}$ are first-order because of (ii). $1 \mathrm{~g}$ As can be seen from the figure, these are precisely the boundaries that were found to be of first order. All others are found to be of second order, with the exceptions of the $\mathrm{M}_{\mathrm{A}}-\mathrm{M}_{\mathrm{B}}$ and $\mathrm{M}_{\mathrm{A}}-\mathrm{M}_{\mathrm{C}}$ boundaries, which form a vertical line at $\beta=3 \pi / 4$ indicated by grey shading in the figure. Along this line, one finds a degenerate minimum connecting $\mathrm{M}_{\mathrm{A}}$ and $\mathrm{M}_{\mathrm{C}}$ phases as shown in Fig. 3(e) for $0.259 \pi<\alpha<0.640 \pi$, and a similar degenerate minimum connecting $\mathrm{M}_{\mathrm{A}}$ and $\mathrm{M}_{\mathrm{B}}$ phases for $0.640 \pi<\alpha<0.740 \pi$. The degenerate behavior can be traced to the fact that $a_{6}=0$ along the line $\beta=3 \pi / 4$. The triple point connecting the $\mathrm{T}, \mathrm{O}$, and $\mathrm{M}_{\mathrm{C}}$ phases at $\alpha=\tan ^{-1}(1 / 3)=0.102 \pi$ and $\beta=\pi$ is also a point at which a degenerate minimum occurs, as shown in Fig. 3(f). The degenerate behaviors are artifacts of the eighth-order truncation, as will be explained more fully in Sec. IVD. Finally, the reader is reminded that because the theory is based on a single polar orientational order parameter, Eq. (3), we do not have the ability to describe transitions to or from the paraelectric $\mathrm{C}$ phase, nor can we describe the more 
complex AFE or AFD phases.

The variation of some physical variable, such as temperature, composition, or pressure, will correspond to a variation of the parameters $\alpha$ and $\beta$ of the model in a way that is not easy to predict a priori. For $\mathrm{BaTiO}_{3}$ and $\mathrm{KNbO}_{3}$, for which the observed phase transition sequence is $\mathrm{R}-\mathrm{O}-\mathrm{T}-\mathrm{C}$ with increasing temperature, it must be the case that the system traverses a roughly vertical trajectory on the phase diagram of Fig. 4, somewhere in the range $\pi<\beta<3 \pi / 2$. (Insofar as eighth-order terms are small for these systems, the trajectory should be near $\beta=\pi$.) The $\mathrm{R}-\mathrm{O}-\mathrm{T}$ sequence can be visualized as traversing Figs. 2(d-f) and 2(a), in that order.

On the other hand, systems such as PZT $\left(\mathrm{PbZr}_{1-x} \mathrm{Ti}_{x} \mathrm{O}_{3}\right)$ that exhibit a morphotropic phase boundary (MPB), i.e., an $\mathrm{R}-\mathrm{T}$ transition as a function of composition $x$, evidently cross the first-order $\mathrm{R}-\mathrm{T}$ phase boundary in the vicinity of $\beta=0$ with increasing $x$. If this trajectory passes to the right of the triple point connecting $\mathrm{R}, \mathrm{T}$, and $\mathrm{M}_{\mathrm{A}}$ phases at $\alpha=\pi / 2$, $\beta=\tan ^{-1}(1 / 3)=0.102 \pi$ in Fig. 4, then the phase transition sequence befomes $\mathrm{R}-\mathrm{M}_{\mathrm{A}}-\mathrm{T}$, as recently observed experimentally 50 . $\mathrm{B}$ The narrowness of the range of $\mathrm{M}_{\mathrm{A}}$ phase, only a few percent in $x$, suggests that the trajectory passes rather close to the triple point. In fact, there are strong experimental indications of the possible existence of a triple point in the $x-7$ phase diagram of PZT near $x \simeq 0.47$ and $T \simeq 100^{\circ} \mathrm{C}$. 5. : 1 Thus, it may be that the behavior near the triple point can be explored experimentally in the PZT system.

Using the topological analysis introduced earlier, we can now clarify the nature of the $\mathrm{T}-\mathrm{M}_{\mathrm{A}}-\mathrm{R}$ transition sequence near the triple point. Referring to Fig. 5, we imagine traversing a downward trajectory of increasing $\alpha$ at fixed $\beta$, slightly to the right of the triple point. Starting deep in the T phase, Fig. 2(a), a saddle point detaches from the $\mathrm{O}$ point and traverses the $\mathrm{M}_{\mathrm{B}}$ line toward the $\mathrm{R}$ point, Fig. 2(b). After it passes through the $\mathrm{R}$ point and emerges on the "other side" (on the $\mathrm{M}_{\mathrm{A}}$ line), we find ourselves in the situation of Fig. 5(a), the R point having been converted to a local minimum. Up to this point, the global minimum remains at $\mathrm{T}$. Next, the $\mathrm{T}$ point converts from a local minimum to a local maximum, with the simultaneous emission of a saddle point along $\mathrm{M}_{\mathrm{C}}$ and a local minimum along $\mathrm{M}_{\mathrm{A}}$, as shown in Fig. 5(b). This event corresponds to the second-order $\mathrm{T}-\mathrm{M}_{\mathrm{A}}$ transition. The first-order $\mathrm{M}_{\mathrm{A}}-\mathrm{R}$ transition then occurs by the crossing of the energies of the local minima of Fig. 5(b). Once in the $\mathrm{R}$ phase, the local minimum and saddle point on the $\mathrm{M}_{\mathrm{A}}$ line annihilate one another to give Fig. 5(c). Finally, deep in the $\mathrm{R}$ phase, the $\mathrm{M}_{\mathrm{C}}$ saddle point eventually arrives at $\mathrm{O}$, giving rise to the situation of Fig. 2(d).

It should thus be emphasized that the present theory makes a definite prediction about the nature of the transitions that occur in the $\mathrm{T}-\mathrm{M}_{\mathrm{A}}-\mathrm{R}$ transition sequence. We can predict that, if it were possible to scan with decreasing $x$ at a temperature below that of the triple point,
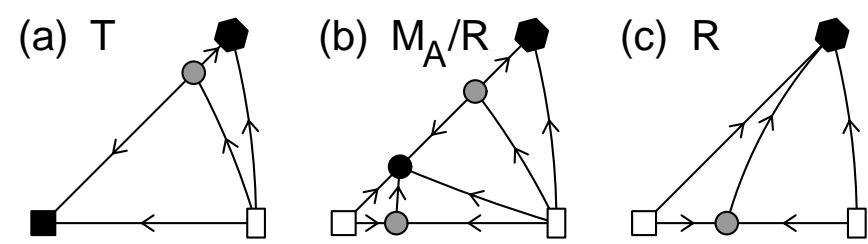

FIG. 5. Topologies encountered in the $\mathrm{T}-\mathrm{M}_{\mathrm{A}}-\mathrm{R}$ transition sequence near the triple point. (a) Topology of $\mathrm{T}$ phase near the second-order $\mathrm{T}-\mathrm{M}_{\mathrm{A}}$ transition. (b) Topology of $\mathrm{M}_{\mathrm{A}}$ phase and, as well, of $\mathrm{R}$ phase near the first-order $\mathrm{M}_{\mathrm{A}}-\mathrm{R}$ transition. (c) Topology occurring deeper in the $\mathrm{R}$ phase.

one would first find a continuous rotation of the polarization from [001] into the (110) plane starting at a critical $x_{c 2}$, and then a discontinuous jump to the [111] direction when there is a crossing of the free energies of the $\mathrm{M}_{\mathrm{A}}$ and $\mathrm{R}$ phases at $x_{c 1}$. Unfortunately, the fact that the $\mathrm{T}-\mathrm{M}_{\mathrm{A}}$ and especially the $\mathrm{R}-\mathrm{M}_{\mathrm{A}}$ boundaries lie almost vertically in the experimental $x-T$ plane may make it difficult to test this prediction, since $x$ can only be varied by preparation of multiple samples. Nevertheless, this scenario seems to be supported by the numerical simulations of Ref. 10.

It is important to note that a triclinic phase does not occur anywhere in the phase diagram of the eighth-order model. However, we do note the possibility of observing new monoclinic phases of types $\mathrm{M}_{\mathrm{B}}$ and $\mathrm{M}_{\mathrm{C}}$ in a region near $\beta \simeq 0.8 \pi$. In fact, the sixth-order model (vertical dotted line at $\beta=\pi$ ) comes very close to yielding a monoclinic $\mathrm{M}_{\mathrm{C}}$ phase near the triple point (grey dot at $\alpha=\tan ^{-1}(3), \beta=\pi$ ) where the $\mathrm{M}_{\mathrm{C}}, \mathrm{T}$, and $\mathrm{O}$ phases are in equilibrium. If a system such as $\mathrm{BaTiO}_{3}$ or $\mathrm{KNbO}_{3}$ could somehow be perturbed so that the variation with temperature would carry the system on a trajectory passing to the left of this triple point in Fig. 4, then a novel $\mathrm{R}-\mathrm{O}-\mathrm{M}_{\mathrm{A}}-\mathrm{T}-\mathrm{C}$ (or even $\mathrm{R}-\mathrm{M}_{\mathrm{B}}-\mathrm{O}-\mathrm{M}_{\mathrm{A}}-\mathrm{T}-\mathrm{C}$ ) transition sequence might be observed. However, to our knowledge, no $\mathrm{M}_{\mathrm{B}}$ or $\mathrm{M}_{\mathrm{C}}$ phase has ever been observed in a cubic perovskite system.

\section{Higher-order expansions}

We have seen that the eighth-order expansion still does not allow for the appearance of a triclinic equilibrium phase for any parameter values. It is natural, then, to ask at what higher order in the expansion a triclinic phase can first occur. The answer is that the expansion must be carried to twelfth order before a triclinic phase can appear.

For, suppose that a triclinic phase is the ground state. Then the minimum of $G$ occurs at a point $\hat{\mathbf{u}}_{0}$ located in the interior of the irreducible wedge, as illustrated in Fig. 3(d). Letting

$$
\begin{aligned}
\widetilde{G}(\hat{\mathbf{u}}) & =G(\hat{\mathbf{u}})-G\left(\hat{\mathbf{u}}_{0}\right), \\
\tilde{g}_{4}(\hat{\mathbf{u}}) & =g_{4}(\hat{\mathbf{u}})-g_{4}\left(\hat{\mathbf{u}}_{0}\right),
\end{aligned}
$$




$$
\tilde{g}_{6}(\hat{\mathbf{u}})=g_{6}(\hat{\mathbf{u}})-g_{6}\left(\hat{\mathbf{u}}_{0}\right),
$$

the expansion Eq. (4) can be rewritten

$$
\widetilde{G}(\hat{\mathbf{u}})=\tilde{a}_{8} \tilde{g}_{4}^{2}+\tilde{a}_{10} \tilde{g}_{4} \tilde{g}_{6}+\tilde{a}_{12} \tilde{g}_{4}^{3}+\tilde{a}_{12}^{\prime} \tilde{g}_{6}^{2}+\ldots,
$$

where the $\tilde{a}_{n}$ are trivial linear combinations of the $a_{n}$. To drop the $\tilde{a}_{4}$ and $\tilde{a}_{6}$ terms, we have used the fact that $G(\hat{\mathbf{u}})$ must be stationary at $\hat{\mathbf{u}}_{0}$. (For this we also need that the gradients of $g_{4}$ and $g_{6}$ never vanish or become parallel at an interior point of the irreducible wedge; this is straightforward to confirm.)

It is now evident that if expansion (13) is truncated at eighth order, then point $\hat{\mathbf{u}}_{0}$ is not an isolated minimum. Instead, it belongs to a degenerate locus of minima corresponding to $\tilde{g}_{4}=0$, i.e., to a contour of the function $g_{4}(\hat{\mathbf{u}})$. This is the situation illustrated in Fig. 3(e). It occurs when $a_{6}=0$, i.e., when $c_{6}+c_{8}=0$, corresponding to the grey vertical line at $\beta=3 \pi / 4$ in Fig. 4 .

If the expansion is carried to tenth order, then it is clear from Eq. (13) that $\widetilde{G}$ still vanishes on this same contour. The two-dimensional Hessian matrix $H_{\mu \nu}=$ $d^{2} G / d \hat{u}_{\mu} d \hat{u}_{\nu}$ then takes the form

$$
H=\left(\begin{array}{ll}
0 & d \\
d & e
\end{array}\right)
$$

where indices $\mu=1$ and 2 correspond to the directions parallel and perpendicular to the $g_{4}$ contour, respectively. $d \neq 0$ as long as $\tilde{a}_{10} \neq 0$, in which case $\operatorname{det} H<0$. Thus, at tenth order, the stationary point $\hat{\mathbf{u}}_{0}$ cannot be a minimum; instead, it is generally an isolated saddle point.

Finally, it is clear that the point $\hat{\mathbf{u}}_{0}$ can be a local minimum if the expansion is carried to twelfth order. For suppose $\tilde{a}_{10}=\tilde{a}_{12}=0$, and $\tilde{a}_{8}$ and $\tilde{a}_{12}^{\prime}$ are positive. Then $\widetilde{G}=\tilde{a}_{8} \tilde{g}_{4}^{2}+\tilde{a}_{12}^{\prime} \tilde{g}_{6}^{2}$ is obviously positive definite, and $\hat{\mathbf{u}}_{0}$ is a true isolated (global) minimum.

Concluding this section, we find that it is necessary to go to surprisingly high order in the free-energy expansion in order to stabilize a ferroelectric state in which there are no symmetry constraints on the order parameter $\mathbf{P}$. Specifically, we find that cubic invariants of twelfth or higher order have to be included to to stabilize such a triclinic phase. We conclude that the discovery (or synthesis) of a material having such behavior may be challenging, but is by no means impossible.

\section{A MICROSCOPIC MODEL}

When structural transitions have some order-disorder character, a model free energy (e.g., (2) or (6) expressed as a function of a macroscopic order parameter (e.g., $\mathbf{u}$ or $\hat{\mathbf{u}}$ ) provides little insight into the local structural fluctuations that underlie the transitions. In such a case, a more appropriate microscopic picture of the high-symmetry phase may be one in which local regions have undergone a symmetry-lowering structural distortion, but in such a way that long-range order has not set in. For example, the description of the phase transition sequence of $\mathrm{BaTiO}_{3}$ and $-\mathrm{KNbO}_{3}$ in terms of the wellknown "eight-site model" 20 assumes the presence of random local rhombohedral displacements in the orthorhombic, tetragonal, and cubic phases. In the present case, Noheda et al. hayeconcluded from their own structural analysis of PZT bohedral PZT, 21 that there may be random local monoclinic displacements which order variously to yield the tetrahedral, rhombohedral, or monoclinic phases near the morphotropic phase boundary. One could then describe the system in terms of fluctuations between minima of a "24-site model."

To make these ideas more precise, suppose that the local displacements of Noheda et al. arise from an optical branch of the phonon spectrum, and let $\mathbf{u}_{l}$ be the vector "local mede amplitude" for that branch within the $l$ th unit cell. 9 Take as a model of the free energy

$$
F[\mathbf{u}]=\sum_{l} f\left(\mathbf{u}_{l}\right)+\frac{1}{2} \sum_{l m}^{\prime} f_{l m}\left(\mathbf{u}_{l}, \mathbf{u}_{m}\right) .
$$

The in-cell energy $f$ is presumed to be strongly nonlinear; the inter-cell coupling $f_{l m}$ may either be bilinear in $\mathbf{u}_{l}$ and $\mathbf{u}_{m}$, or of higher order. $f$ is also presumed to dominate the $f_{l m}$ so that, to a good approximation, its global minima establish the possible magnitude and orientations $\mathbf{u}_{l}^{\alpha}$ of $\mathbf{u}_{l} . f\left(\mathbf{u}_{l}\right)$ can be subjected to precisely the same methods of analysis as applied to $F(\mathbf{u})$ in Secs. II $[\mathrm{IV}$ above, with parallel results. The $\mathbf{u}_{l}^{\alpha}$ so obtained can then be substituted into $F$ in Eq. (15), yielding.

$$
F[\alpha]=F_{0}+\frac{1}{2} \sum_{l m}^{\prime} f_{l m}\left(\mathbf{u}_{l}^{\alpha}, \mathbf{u}_{m}^{\beta}\right) .
$$

This can be regarded as a 24 -site version of the $q$-state Potts model,

A statistical analysis can be carried out for various forms of $f_{l m}$ (e.g., bilinear) to capture the cubic (completely disordered), tetragonal (partially disordered), rhombohedral (partially disordered), and monoclinic (fully ordered) phases of the case where $\mathbf{u}_{l}^{\alpha}$ takes on all of the 24 symmetrically equivalent monoclinic displacements. This type of analysis has already been carried out for orientational order-disorder transitions and plastic crystals. 22

\section{SUMMARY AND CONCLUSIONS}

The original Devonshire theoryt gave a natural explanation for the appearance of tetragonal, orthorhombic, and rhombohedral phases in materials such as $\mathrm{BaTiO}_{3}$ based on a sixth-order free-energy expansion. Here, we have confirmed that these ferroelectric phases, in which the order parameter $\mathbf{P}$ is confined to a symmetry axis, 
are the only ones permitted by the sixth-order version of the theory. Moreover, we have clarified the nature of the phases that may be expected to appear at higher orders in the expansion. In particular, we have shown that the extension of the theory to eighth order allows one to describe, in addition, three kinds of monoclinic phase in which $\mathbf{P}$ is confined only to a symmetry plane. To obtain a triclinic phase in which $\mathbf{P}$ is unconstrained by symmetry, we have shown that a twelfth-order version of the theory is needed. A topological analysis of the critical points of the energy surface has been used to facilitate the discussion of the relevant phases and phase transitions.

The present theory may provide some added insight into the phase behavior of conventional ferroelectrics such as $\mathrm{BaTiO}_{3}$, but the principal new results concern cases in which the eighth-order terms are important. In particular, the theory provides a natural explanation for the monoclipif $\mathrm{M}_{\mathrm{A}}$ phase recently observed experimentally in PZT 5.8 It also predicts that if a triple point of equilibrium between $\mathrm{T}, \mathrm{R}$, and $\mathrm{M}_{\mathrm{A}}$ phases occurs, then it will be one at which first-order $\mathrm{R}-\mathrm{T}$ and $\mathrm{R}-\mathrm{M}_{\mathrm{A}}$ boundaries meet a second-order $\mathrm{T}-\mathrm{M}_{\mathrm{A}}$ boundary. That is, $\mathbf{P}$ will rotate into the $(1 \overline{1} 0)$ mirror plane continuously from the $\mathrm{T}$ side but discontinuously from the $\mathrm{R}$ side.

Noheda et al 8 have shown such a triple point in their Fig. 6, in agreement with our analysis. However, they also show a region of coexistence of the $\mathrm{T}$ and $\mathrm{M}_{\mathrm{A}}$ phases in the same phase diagram. In a homogeneous sample, such a coexistence region can be due to hysteresis arising from nucleation barriers to a first-order phase transition. The eighth-order theory predicts the $\mathrm{T}-\mathrm{M}_{\mathrm{A}}$ phase boundary to be of second order, in which case there can be no hysteresis or nucleation barriers. In principle, higher-order contributions to the free energy could be large enough to change the order of the $\mathrm{T}-\mathrm{M}_{\mathrm{A}}$ transition. However, a more likely explanation is that the samples studied by Noheda et al. may be inhomogeneous. We note that these authors did not report hysteresis but did report a two-phase coexistence region near the R-T boundary between 500 and $575 \mathrm{~K}$ in Fig. 5 of Ref. \& Attributing this to inhomogeneity, we estimate that the concentration variation may be of order $1 \%$ from the slope of the $\mathrm{R}-\mathrm{T} / \mathrm{M}-\mathrm{T}$ phase boundary in Fig. 6 of Ref. 8. Composition inhomogeneity of that magnitude would be sufficient to account for the $\mathrm{T}-\mathrm{M}_{\mathrm{A}}$ phase coexistence shown in that figure.

Finally, the work may provide some guidance in the search for even more novel $\mathrm{M}_{\mathrm{C}}, \mathrm{M}_{\mathrm{B}}$, and triclinic ferroelectric phases. It also may be of utility in other kinds of cubic systems with other kinds of vector order parameters, e.g., ferromagnetic systems.

\section{ACKNOWLEDGMENTS}

D.V. acknowledges support of ONR Grant N0001497-1-0048. We wish to thank L. Bellaiche, A. García, A. Khachaturyan, and B. Noheda for useful comments and discussions.

\section{APPENDIX A: DETAILS OF SIXTH-ORDER THEORY}

The purpose of this Appendix is to give further details about the sequence of transitions that occurs in the sixthorder model of Sec. IV B. Recall that the behavior in this model is governed by a single dimensionless parameter $\alpha$ defined via Eq. (10).

There are ten critical values of $\alpha$ that we can define as

$$
\begin{aligned}
\alpha_{1} & =-0.3976 \pi=-\tan ^{-1}(3) \\
\alpha_{2} & =0.2256 \pi=\tan ^{-1}(6 / 7) \\
\alpha_{3} & =0.25 \pi \\
\alpha_{4} & =0.3128 \pi=\tan ^{-1}(3 / 2) \\
\alpha_{5} & =0.5 \pi \\
\alpha_{6} & =0.6024 \pi=\pi-\tan ^{-1}(3) \\
\alpha_{7} & =1.2256 \pi=\pi+\tan ^{-1}(6 / 7) \\
\alpha_{8} & =1.25 \pi \\
\alpha_{9} & =1.3128 \pi=\pi+\tan ^{-1}(3 / 2) \\
\alpha_{10} & =1.5 \pi
\end{aligned}
$$

The sequence of phases can be followed on Fig. 4 by tracing the vertical dotted lines, first from top to bottom at $\beta=0$ for $0<\alpha<\pi$, and then from bottom to top at $\beta=\pi$ for $\pi<\alpha<2 \pi$.

In the $\mathrm{T}$ phase, the system exhibits the topology of Fig. 2(a) for $\alpha_{1}<\alpha<\alpha_{2}$; Fig. 2(b) for $\alpha_{2}<\alpha<$ $\alpha_{4}$; and Fig. 2(c) for $\alpha_{4}<\alpha<\alpha_{5}$. At $\alpha_{2}$, point $\mathrm{O}$ converts to a local maximum and simultaneously a saddle point appears along the $\mathrm{M}_{\mathrm{B}}$ symmetry line. There is an irrelevant crossing of the $\mathrm{R}$ and $\mathrm{O}$ maxima at $\alpha_{3}$. At $\alpha_{4}, \mathrm{R}$ becomes a local minimum, and the saddle point switches from the $\mathrm{M}_{\mathrm{B}}$ to the $\mathrm{M}_{\mathrm{A}}$ symmetry line. The transition from $\mathrm{T}$ to $\mathrm{R}$ is first order at $\alpha_{5}$.

The system falls into an $\mathrm{R}$ ground state corresponding to Fig. 2(c) for $\alpha_{5}<\alpha<\alpha_{6}$; Fig. 2(d) for $\alpha_{6}<\alpha<\alpha_{7}$; and Fig. 2(e) for $\alpha_{7}<\alpha<\alpha_{8}$. The $\mathrm{M}_{\mathrm{A}}$ saddle point disappears and $\mathrm{T}$ is converted to a maximum at $\alpha_{6}$, and a new $\mathrm{M}_{\mathrm{B}}$ saddle point emerges with the conversion of $\mathrm{O}$ to a local minimum at $\alpha_{7}$. The transition from $\mathrm{R}$ to $\mathrm{O}$ is first order at $\alpha_{8}$.

Finally, an $\mathrm{O}$ phases occurs as illustrated in Fig. 2(e) for $\alpha_{8}<\alpha<\alpha_{9}$, and as in Fig. 2(f) for $\alpha_{9}<\alpha<\alpha_{1}+2 \pi$. The saddle point at $\mathrm{M}_{\mathrm{B}}$ vanishes and $\mathrm{R}$ is converted to a local maximum at $\alpha_{9}$. There is an irrelevant crossing of the $\mathrm{T}$ and $\mathrm{R}$ maxima at $\alpha_{10}$. The transition from $\mathrm{O}$ to $\mathrm{T}$ at $1.6024 \pi$ is singular, in that the energy surface becomes exactly flat along the entire $\mathrm{M}_{\mathrm{C}}$ symmetry line. 
${ }^{1}$ A.F. Devonshire, Phil. Mag. 40, 1040 (1949).

${ }^{2}$ A.F. Devonshire, Phil. Mag. 42, 1065 (1951); Adv. Phys. 3, 85 (1954).

${ }^{3}$ B. Jaffe, W.R. Cook, and H. Jaffe, Piezoelectric Ceramics (Academic Press, London, 1971).

${ }^{4}$ M.J. Haun, E. Furman, S.J. Jang, and L.E. Cross, Ferroelectrics 99, 13 (1989).

${ }^{5}$ B. Noheda, D.E. Cox, G. Shirane, J.A. Gonzalo, L.E. Cross, and S.-E. Park, Appl. Phys. Lett. 74, 2059 (1999).

${ }^{6}$ R. Guo, L.E. Cross, S-E. Park, B. Noheda, D.E. Cox and G. Shirane, Phys. Rev. Lett. 84, 5423 (2000).

${ }^{7}$ B. Noheda, J.A. Gonzalo, L.E. Cross, R. Guo, S.-E. Park, D.E. Cox, and G. Shirane, Phys. Rev. B 61, 8687 (2000).

${ }^{8}$ B. Noheda, D.E. Cox, G. Shirane, R. Guo, B. Jones, and L.E. Cross, Phys. Rev. B, in press (preprint http://arXiv.org/abs/cond-mat/0006152).

9 W. Zhong, D. Vanderbilt, and K.M. Rabe, Phys. Rev. B 52, 6301 (1995).

${ }^{10}$ L. Bellaiche, A. García, and D. Vanderbilt, Phys. Rev. Lett. 84, 5427 (2000).

11 A.G. Souza Filho et al., Phys. Rev. B 61, 14283 (2000).

${ }^{12}$ Eq. (3) of Ref. 11 implies that the shear elastic constant vanishes at $T=T_{M}$ even under conditions of fixed polarization $\mathbf{P}$. Thus, the primary order parameter of the transition is strain, not polarization, and the transition is to an "improper" or "extrinsic" ferroelectric state.

${ }^{13}$ For this to be so, the equilibrium magnitude $|\mathbf{u}|$ for a given $\hat{\mathbf{u}}$ should not be too a strong function of $\hat{\mathbf{u}}$.

${ }^{14}$ F.C. Von der Lage and H.A. Bethe, Phys. Rev. 71, 612 (1947).

15 A. García, private communication.

${ }^{16}$ M. Morse, Functional Topology and Abstract Variational Theory, Memoires Sciences Mathematiques (GauthierVillars, Paris, 1938), Fascicule 92; H. Seifert and W. Threlfall, Variationsrechnung Im Grossen, Hamburger Mathematische Einzelschriften (B.G. Teubner, Leipsig und Berlin, 1938), Vol. 24.

17 J.C. Phillips, Phys. Rev. 104, 1263 (1956).

${ }^{18}$ L.D. Landau and E.M. Lifshitz, Statistical Physics, Part 1 (3rd Edition) (Pergamon Press, New York, 1980).

19 Representing $\hat{\mathbf{u}}$ by polar coordinates $(\theta, \phi)$ measured with respect to the (111) axis, a function such as $\sin ^{3}(\theta) \cos (3 \phi)$ is third order in displacements from $\theta=0$ and is invariant with respect to the rhombohedral symmetry group.

${ }^{20}$ R. Comes, M. Lambert, and A. Guinier, C.R. Acad. Sci. Paris 226, 959 (1968); Solid State Comm. 6, 715 (1968); J. P. Sokoloff, L. L. Chase, and D. Rytz, Phys. Rev. B 38, 597 (1988).

${ }^{21}$ D.L. Corker, A.M. Glazer, R.W. Whatmore, A. Stallard, and F. Fauth, J. Phys. Cond. Matt. 10, 6251 (1998).

${ }^{22}$ W. Breymann and R.M. Pick, Europhys. Lett. 6, 227 (1988). 